\title{
Effect of annular secondary conductor in a linear electromagnetic stirrer
}

\author{
R MADHAVAN $^{1}$ and V RAMANARAYANAN ${ }^{2}$ \\ ${ }^{1}$ Department of Electrical Engineering, Indian Institute of Technology, Bombay, \\ Powai, Mumbai 400076 \\ ${ }^{2}$ Power Electronics Group, Department of Electrical Engineering, Indian Institute \\ of Science, Bangalore 560012 \\ e-mail: madhavan@ee.iitb.ac.in; vram@ee.iisc.ernet.in
}

\begin{abstract}
This paper presents the variation of average axial force density in the annular secondary conductor of a linear electromagnetic stirrer. Different geometries of secondaries are considered for numerical and experimental validation namely, 1 . hollow annular ring, 2. annular ring with a solid cylinder and 3 . solid cylinder. Experimental and numerical simulations are performed for a 2-pole in house built $15 \mathrm{~kW}$ linear electromagnetic stirrer (EMS). It is observed for a supply current of $200 \mathrm{~A}$ at $30 \mathrm{~Hz}$ the force densities in the hollow annular ring is $67 \%$ higher than the equivalent solid cylinder. The same values are $33 \%$ for annular ring with a solid cylinder. Force density variation with supply frequency and current are also reported. Numerical simulations using finite element model are validated with experimental results.
\end{abstract}

Keywords. Linear electromagnetic stirrer; finite element analysis; and annular ring.

\section{Introduction}

Electromagnetic stirrers (EMS) are used to stir the molten metal. The main advantage of these devices is the absence of any physical contact between the material and the stirring device. Two types of EMS are commonly used in practice: The linear stirrer and the rotary stirrer. A linear stirrer operates basically the same way as a linear induction motor. An a.c. current flows through the coil of the primary and generates an eddy current in the secondary conductor. The eddy current in the secondary conductor interacts with the imposed magnetic fields to produce the Lorentz's force in the secondary. The numerical model of the travelling field in the secondary conductor was analysed by Fireteanu \& Gheysens (1992), and the heating and driving effects of the electromagnetic field were also studied. The influence of the excitation frequency and current on the axial force was discussed for a $2 \mathrm{~kW}$ linear EMS in (Milind \& Ramanarayanan 2004).

Pumping (or) stirring liquid metals in metallurgical and nuclear power applications requires construction simplicity, absence of moving parts, perfect sealing, and easy maintenance 


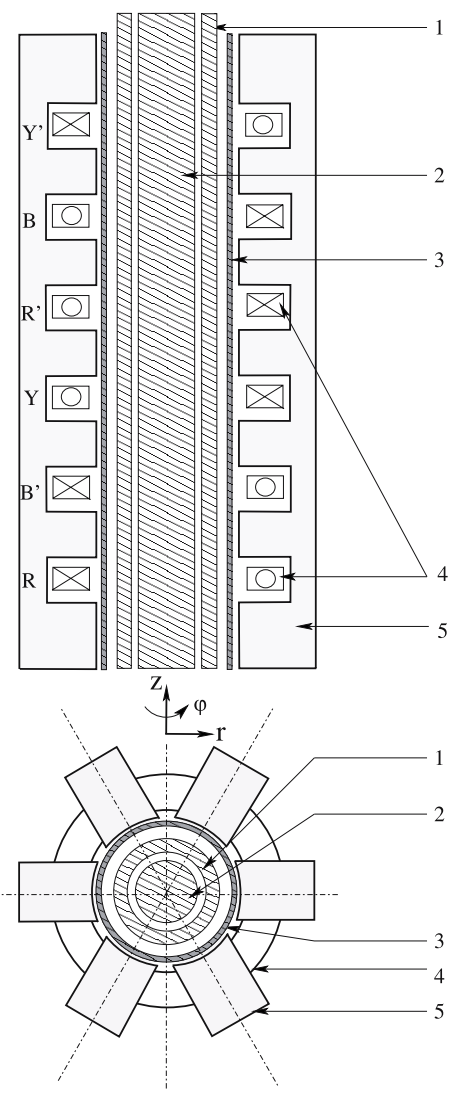

(a)

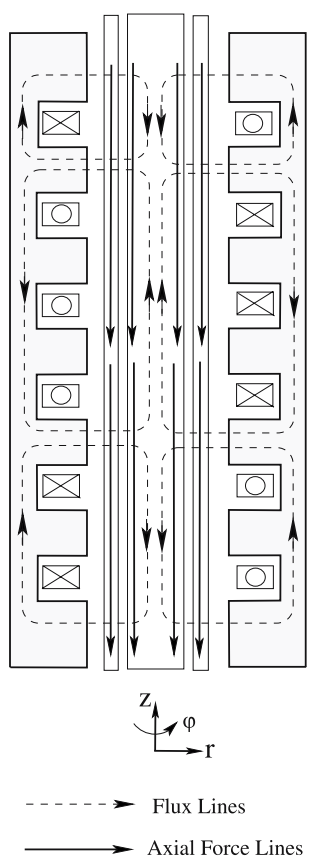

(b)
Figure 1. (a) Basic model of a linear EMS, [1. Secondary annular ring, 2. Secondary solid cylinder, 3. Vacuum chamber, 4. Primary circular coils, 5. Primary magnetic core], (b) Flux and axial force lines.

(Boldea \& Nasar 1985). These requirements can be easily satisfied by using annular linear electromagnetic pumps (ALIP). The main objective of this paper is to study the variation of axial force density using aluminum annular ring as secondary mold. Hollow annular ring, annular ring with a solid cylinder and solid cylinder having the same outer diameter as that of annular ring are considered for numerical and experimental validation.

Figure 1 shows a basic model of a linear electromagnetic stirrer with a multi-turn single layer primary and a single turn short circuited secondary conductor, separated by a small airgap. When an alternating current flows in the primary, voltages are induced in the secondary conductor which cause eddy currents to flow in it. The eddy current and the resultant net magnetic field interact to give radial and axial mechanical forces. It is seen that the radial forces average to zero whereas the axial forces average in the direction of supply phase sequence resulting in movement of the secondary conductor.

The equations governing the electromagnetic field are given by,

Ampere's Law: $\nabla \times H=J$.

Faraday's Law: $\nabla \times E=-\frac{\partial B}{\partial t}$.

Ohm's Law: $J=\sigma E$. 


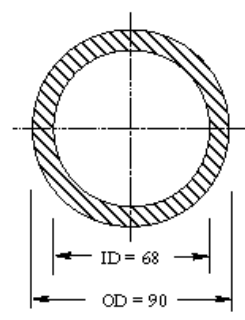

(a)

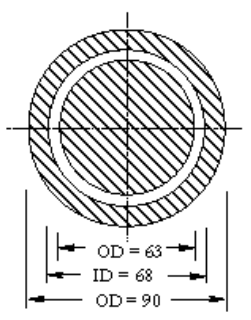

(b)

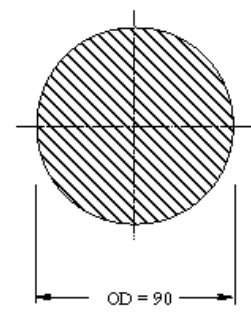

(c)
Figure 2. Different types of secondaries (values in mm), (a) Hollow annular ring, (b) Annular ring with a solid cylinder, (c) Solid cylinder.

Mechanical forces in the secondary are given by,

$$
\begin{aligned}
& \text { Axial force density: } \overrightarrow{F_{z}}=\overrightarrow{J_{\varphi}} \times \overrightarrow{B_{r}} \text {. } \\
& \text { Radial force density: } \overrightarrow{F_{r}}=\overrightarrow{J_{\varphi}} \times \overrightarrow{B_{z}},
\end{aligned}
$$

where $\mathrm{E}$ is the electric field intensity in $\mathrm{V} / \mathrm{m}, \mathrm{H}$ is the magnetic field intensity in $\mathrm{A} / \mathrm{m}, J_{\varphi}$ is the induced eddy current density in $\mathrm{A} / \mathrm{m}^{2}$ along $\varphi$ direction, $B_{r}$ is the radial magnetic flux density and $B_{z}$ is the axial magnetic flux density expressed in Tesla. Closed form solution is not available for linear EMS. Series solution giving approximate results are available in Warren (1942); Chari \& Salon (2000).

Figure 2 shows the details of secondaries used in the numerical simulation and experimental validation. All cylinders have an equal length (1) of $310 \mathrm{~mm}$.

\section{Finite element analysis}

The geometrical and property data used in simulation for the MagNet software model is presented in table 1. Mesh independence studies are conducted prior to the final simulation results presented here. It was found that maximum element size value must be half of the skin depth value to give sufficiently accurate results comparable with experimentally obtained value. Simulations are carried out by varying the current, frequency and diameter for individual

Table 1. Stirrer design parameters.

\begin{tabular}{ll}
\hline Bore Diameter D & $0 \cdot 140 \mathrm{~m}$ \\
Stirrer length L & $0 \cdot 284 \mathrm{~m}$ \\
Pole pitch $\tau$ & $0 \cdot 117 \mathrm{~m}$ \\
Slot pitch $\tau_{s}$ & $0 \cdot 04 \mathrm{~m}$ \\
Excitation current I & 50 A to 200 A R.M.S \\
$\mu_{r}$ of Aluminum & 1 \\
$\sigma$ for Aluminum at $20^{\circ} \mathrm{C}$ & $37.4 * 10^{6} \mathrm{~S}-\mathrm{m}^{-1}$ \\
Number of slots per pole and per phase & 1 \\
Velocity of the travelling wave at $50 \mathrm{~Hz}$ & $11.7 \mathrm{~m} / \mathrm{sec}$ \\
Depth of penetration of flux at $50 \mathrm{~Hz}$ & $12 \mathrm{~mm}$ \\
Secondary element size at $50 \mathrm{~Hz}$ & $6 \mathrm{~mm}$ \\
Secondary mesh density at $50 \mathrm{~Hz}$ & $40 * 10^{-3}$ elements $/ \mathrm{mm}^{3}$
\end{tabular}




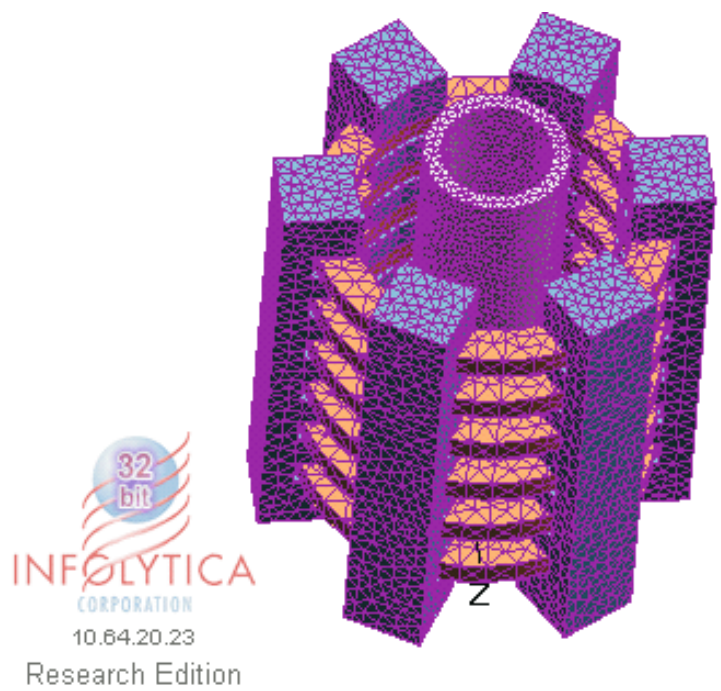

Figure 3. 3-D mesh model of annular linear EMS.

case. Time-harmonic 3-D solver and tetrahedral mesh type is used for solving the model. Figure 3 shows the 3D mesh model with a hollow annular ring as a secondary and also the placement of primary poles in the linear EMS is shown in the model.

\section{Experimental procedure}

Figure 4 shows the prototype of 2-pole linear electromagnetic stirrer, used for experiments. The primary coil is constructed of copper and has a radius of $170 \mathrm{~mm}$, and with 10 turns. The coils are water cooled to keep the operating temperature low. Core is made up of magnetic steel and coated with an anti rust varnish.

Figure 5 shows the different types of secondaries (aluminum cores) used for the experimental validation. All the cores are made up of Al356. Hooks are attached with the secondaries to the weighing balance to measure the force.

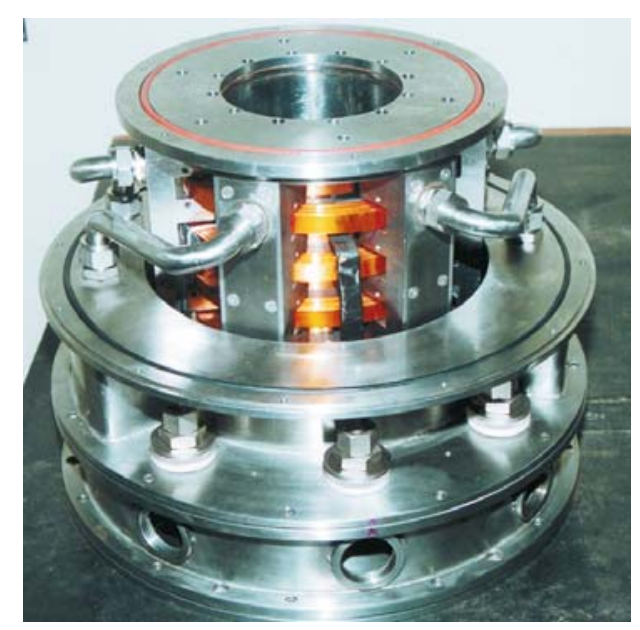

Figure 4. Prototype of 2-pole stirrer. 

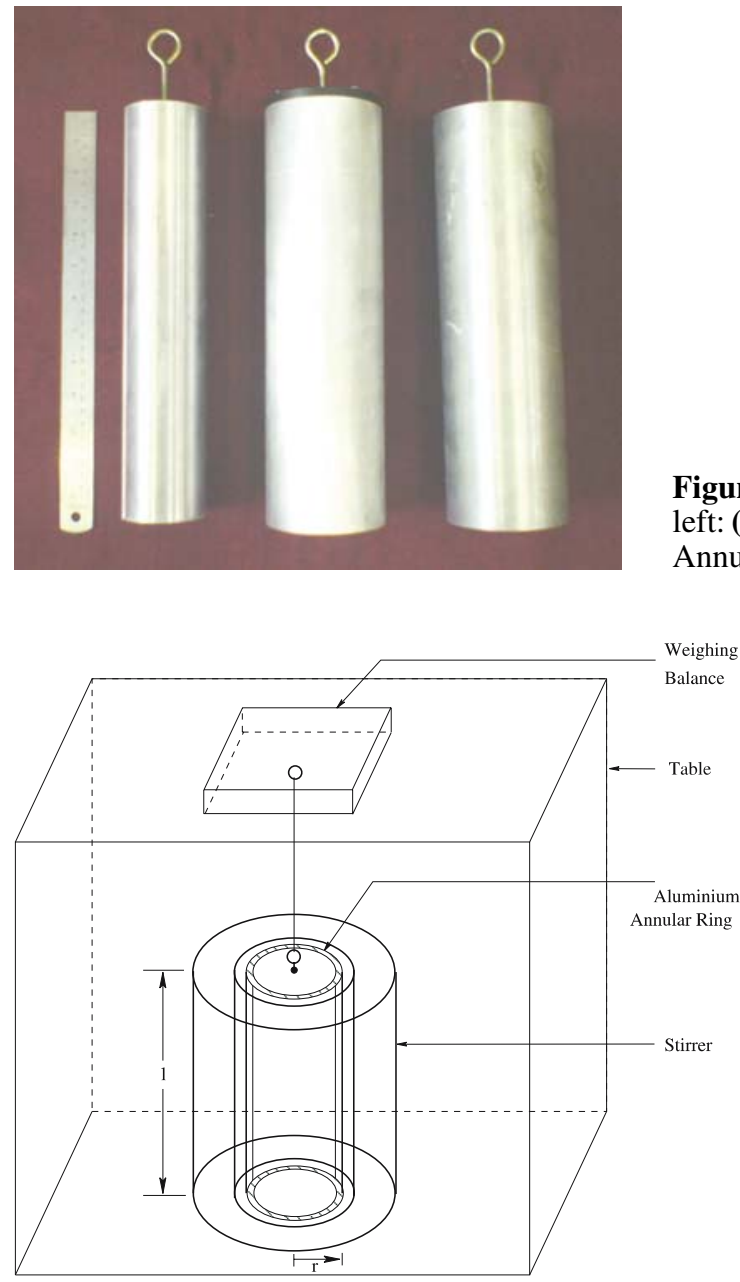

Figure 5. Experimental secondaries [from right to left: (a) Solid cylinder $(O D=63 \mathrm{~mm})$, (b) Hollow Annular ring, (c) Solid cylinder $(O D=90 \mathrm{~mm})]$.

Figure 6. Experimental testing table.

Figure 6 shows the schematic of the experimental set-up used for measuring force. A weighing balance is used to measure the change in weight in the aluminum cylinder placed inside the axially annular space of the stirrer to form the secondary.

Figure 7 shows the block diagram of power supply to EMS. An autotransformer is used for reduced voltage starting of the system. Behaviour of the system at start-up is not of much

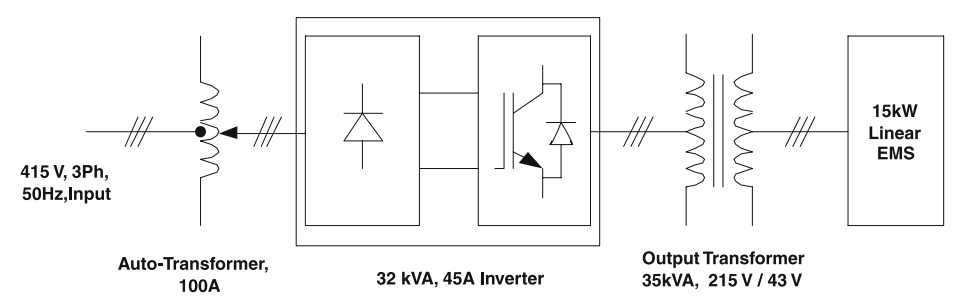

Figure 7. Block diagram of EMS set-up. 


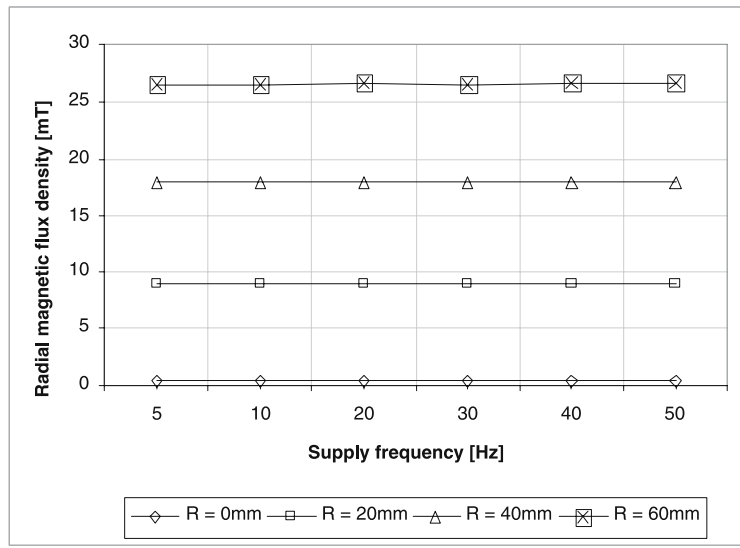

Figure 8. Numerical variation of radial flux density along the radius.

concern as the stirrer is being driven from a voltage source inverter (VSI) coupled with output step down transformer. The constant volts per hertz (V/F) control is used in the VSI. Output frequency of the inverter is controlled automatically through a programmable logic controller (PLC) system.

\section{Results and discussion}

It is important to understand the effect of annular type secondary with other types of secondaries. So, this section details the comparative analysis of numerical and experimental results.

\subsection{Simulation results}

Simulation results show the variation of radial flux density along the radius $(I=200 \mathrm{~A}, L=$ $142 \mathrm{~mm}$ ) of the secondary at different frequencies and this is shown in figure 8. It is concluded that flux density is constant for different frequencies and it varies proportionally with radius. The variation of axial flux density along the length $(I=200 \mathrm{~A}, R=60 \mathrm{~mm})$ of the stirrer at different frequencies is shown in figure 9.

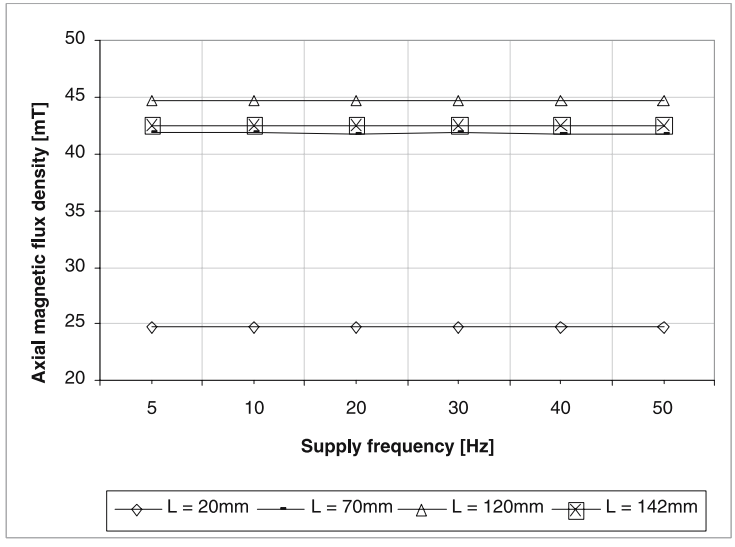

Figure 9. Numerical variation of axial flux density along the length. 


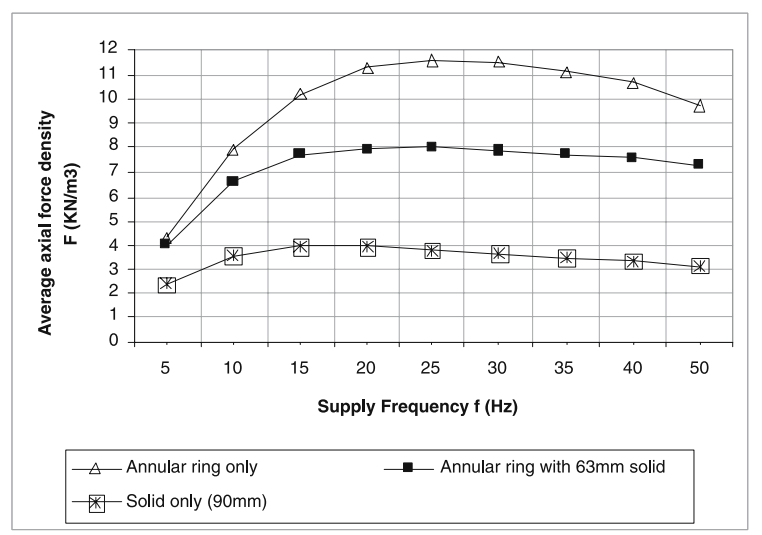

Figure 10. Numerical variation of axial force density with frequencies.

The variation of average axial force density with different frequencies is shown in figure 10 . It is observed that for a supply current of $200 \mathrm{~A}$, the force density in the annular ring is higher than other secondaries. Further, the induced eddy current density is concentrated within volume of the annular ring as shown in figure 11. Since there is no eddy current effect in the airgap, a linear relation between the flux and the depth of penetration can be obtained. While in the aluminum secondary, the induced eddy currents will produce a flux that counters the flux generated by the primary currents. The resultant flux can be represented by a non-linear function (Cheng-Tsung Liu 2007; Davies 1990). It is interesting to note that although the force density differs for the different secondaries, the maximum force density coincides at a $f=25 \mathrm{~Hz}$. The variation of average axial force density with different supply current for a frequency of $25 \mathrm{~Hz}$ is shown in figure 12. It is observed that the force density is proportional to square of the current for both annular ring and solid cylinder secondaries.

\subsection{Experimental results}

The variation of radial and axial component of the flux density in the airgap was measured using gauss meter. Tangential and axial hall probes were used for measuring the radial and axial field respectively. Figure 13 shows the variation of radial flux density against frequency,

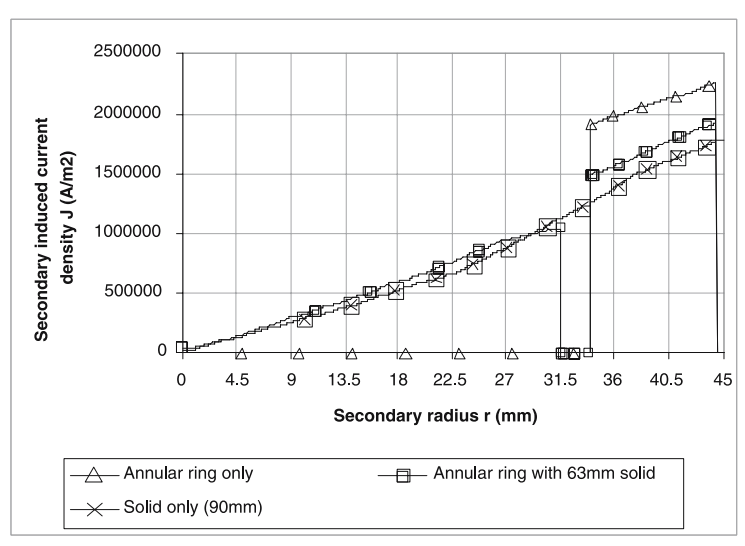

Figure 11. Numerical variation of induced eddy current density with radius. 

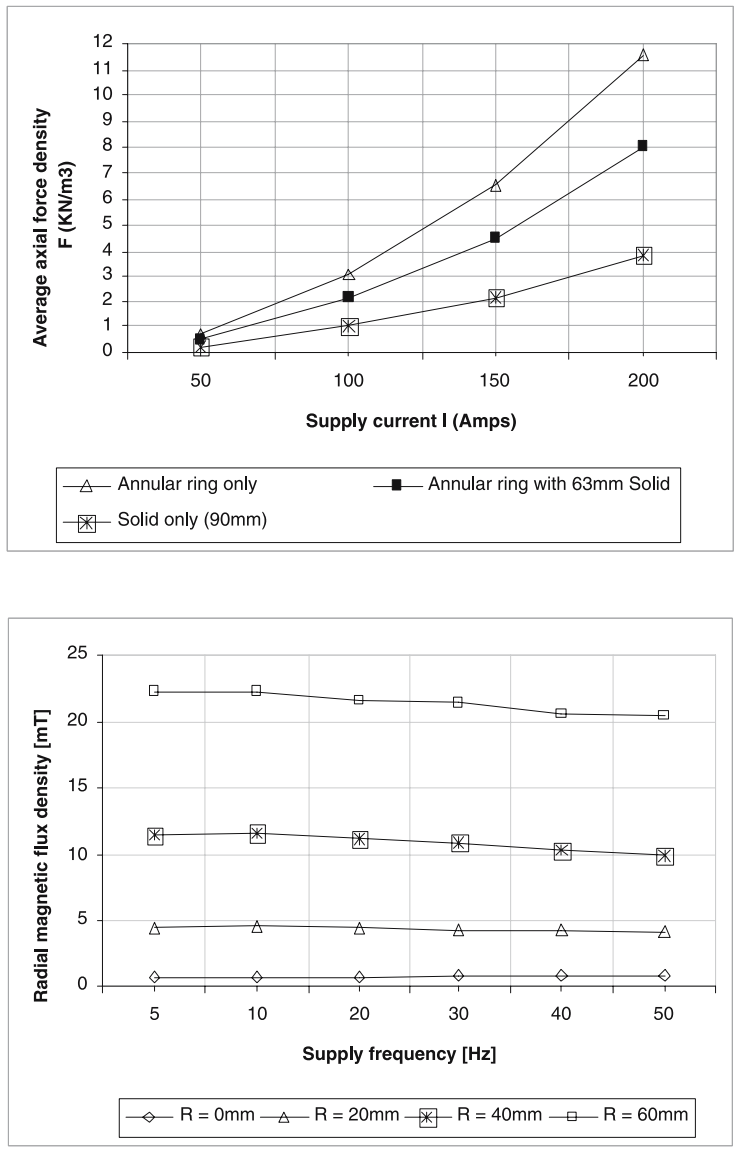

Figure 12. Numerical variation of axial force density with supply current.

Figure 13. Experimental variation of radial flux density along the radius.

measured at different radial positions $(I=200 \mathrm{~A}, L=142 \mathrm{~mm})$. It is observed that radial field increased from the center point to the outer surface. Figure 14 shows the variation of axial flux density against frequency, measured at different positions along the length $(I=$ $200 \mathrm{~A}, R=60 \mathrm{~mm}$ ). It is observed that axial field is pronounced at the center.

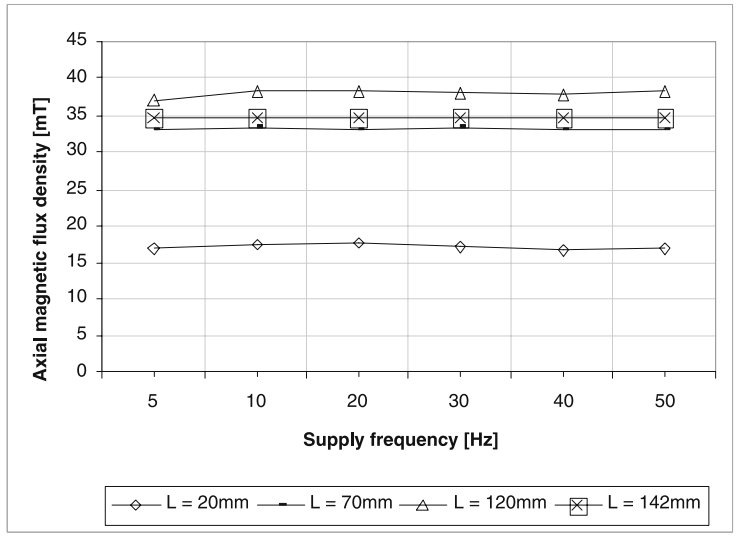

Figure 14. Experimental variation of axial flux density along the length. 


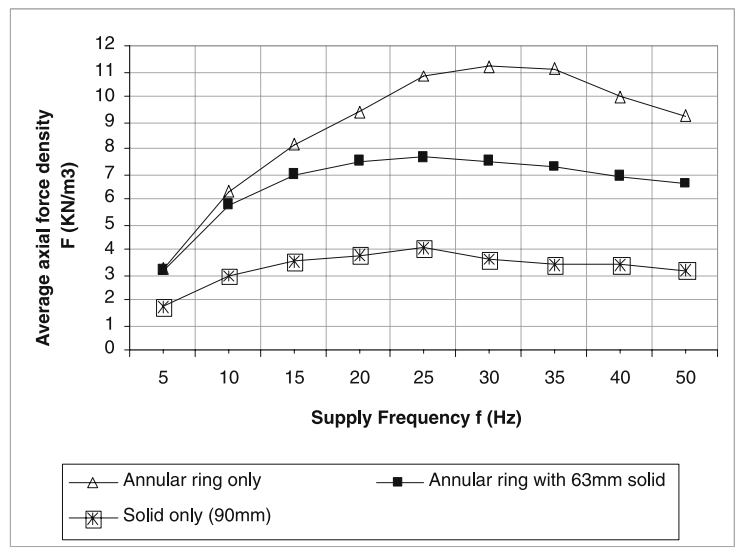

Figure 15. Experimental variation of axial force density with frequencies.

Experimental results show the variation of average axial force density with different frequencies in figure 15. It is observed that annular ring secondary force density is higher at $30 \mathrm{~Hz}$. The variation of average axial force density with different supply current for a frequency of $25 \mathrm{~Hz}$ is shown in figure 16.

The following results are obtained through the numerical and experimental values.

(i) For a fixed current of $200 \mathrm{~A}$, the annular ring secondary force density is more than solid secondary force density. This is on account of the fact that the annular ring has less volume.

(ii) When the solid secondary is inserted into the annular ring, it is observed that annular ring force density is decreased. This can be explained by the fact that the addition of inner aluminum core reduces the eddy current in the annular ring.

(iii) When the diameter of the secondary increases, the maximum force density in the secondary occurs at low frequency. This is on account of skin effect.

(iv) At lower diameter of secondary, the coupling effect is more than the depth of penetration.

(v) When the diameter of the solid secondary inside the annular ring increases, the force density in the annular ring is decreased.

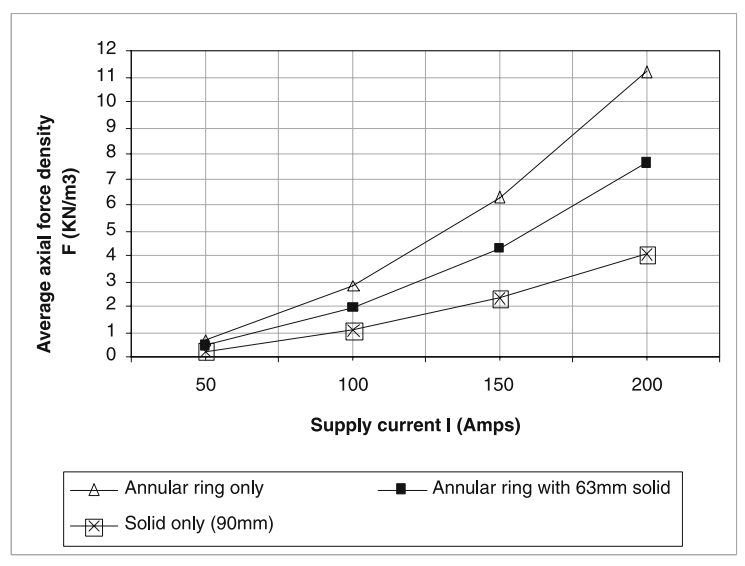

Figure 16. Experimental variation of axial force density with supply current. 


\section{Conclusion}

By replacing the solid conducting annular secondary of linear electromagnetic stirrer with a liquid metal, we obtain a linear motion magneto-hydrodynamic (MHD) machine. The linear induction MHD machines are used in metallurgy and nuclear power plants as liquid-metal pumps. The results demonstrated in this paper is useful for designing annular linear induction pump (ALIP) application. This paper detailed about the usefulness of the different type of secondaries for linear electromagnetic stirrer. It gives good insight of distribution of magnetic field density inside the airgap. Experimental results are showed the validity of the numerical simulation.

The Experiments were conducted at National Facility for Semi Solid Forming under a generous grant from Ministry of Steel and Mines, Department of Science and Technology (DST), Defence Research Development Organisation (DRDO). The help and support provided by the funding agencies and Prof. K S S Murthy, Prof. Pradip Dutta and Pramod Kumar (Ph.D student) of Mechanical Engineering Department is gratefully acknowledged.

\section{References}

Boldea I Nasar S A 1985 Linear motion electromagnetic systems New York: John Wiley and Sons Chari M V K, Sheppard J Salon 2000 Numerical methods in electromagnetism Academic Press

Cheng-Tsung Liu 2007 Analytical model developments and field verifications of a linear Inductiontype electromagnetic stirrer. Proceedings of IEEE-IEMDC 381-385

Davies E J 1990 Conduction and induction heating, IEE Power Engineering Series II. London, UK: Peter Peregrinus Ltd

Fireteanu V Gheysens R 1992 Numerical modelling of the traveling field diffusion, induction heating and electromagnetic stirring. IEEE Trans. Magnetics 28(2) 1489-1492

Milind S, Ramanarayanan V 2004 Design and analysis of a linear type electromagnetic stirrer. Proceedings of IEEE-IAS 188-194

Warren A G 1942 Mathematics applied to electrical engineering Chapman and Hall

MagNet 3-D FEM Software, INFOLYTICA CORPORATION, Canada (www.infolytica.com) 